\title{
First-arrival traveltime tomography for anisotropic media using the adjoint-state method
}

\author{
Umair bin Waheed ${ }^{1}$, Garret Flagg ${ }^{2}$, and Can Evren Yarman ${ }^{3}$
}

\begin{abstract}
Traveltime tomography using transmission data has been widely used for static corrections and for obtaining near-surface models for seismic depth imaging. More recently, it is also being used to build initial models for full-waveform inversion. The classic traveltime tomography approach based on ray tracing has difficulties in handling large data sets arising from current seismic acquisition surveys. Some of these difficulties can be addressed using the adjoint-state method, due to its low memory requirement and numerical efficiency. By coupling the gradient computation to nonlinear optimization, it avoids the need for explicit computation of the Fréchet derivative matrix. Furthermore, its cost is equivalent to twice the solution of the forward-
\end{abstract}

modeling problem, irrespective of the size of the input data. The presence of anisotropy in the subsurface has been well established during the past few decades. The improved seismic images obtained by incorporating anisotropy into the seismic processing workflow justify the effort. However, previous literature on the adjoint-state method has only addressed the isotropic approximation of the subsurface. We have extended the adjointstate technique for first-arrival traveltime tomography to vertical transversely isotropic (VTI) media. Because $\delta$ is weakly resolvable from surface seismic alone, we have developed the mathematical framework and procedure to invert for $v_{\mathrm{NMO}}$ and $\eta$. Our numerical tests on the VTI SEAM model demonstrate the ability of the algorithm to invert for near-surface model parameters and reveal the accuracy achievable by the algorithm.

\section{INTRODUCTION}

First-arrival traveltime (FAT) tomography has been used for nearsurface modeling, which has been used for static corrections in complex media (Marsden, 1993; Bergman et al., 2004), as well as an initial model for depth migration (Dessa et al., 2004) and fullwaveform inversion (FWI) (Brenders and Pratt, 2007; Virieux and Operto, 2009). FAT tomography is composed of two parts, namely, the forward modeling and the inversion. In forward modeling, given a background medium, the FATs are computed by solving the corresponding eikonal equation. The inversion part aims to find the medium parameters that minimize the difference between the measurements and the modeled FATs. The difference between the traveltimes is measured by a cost function.

Minimization of the cost function is a nonlinear optimization problem. In the classical approach of ray-based tomography, it is solved iteratively by linearization of the tomography operator. At each iteration, the forward-modeling operator is linearized with respect to the initial medium parameters. Then, the linearized tomography problem is solved to compute the update on the initial model parameters. Linearization of the tomography operator requires computation of the Fréchet derivative of the forward-modeling operator, which can be explicitly computed by using the ray equations coming from the solution of the eikonal equation. Then, the linearized tomography operator can be inverted iteratively (Zelt and Barton, 1998; Woodward et al., 2008). The cost of computation and storage of the linearized tomography operator increases with the amount of available data, as well as the number of medium parameters.

Alternatively, the nonlinear optimization problem can be combined with the adjoint-state method to avoid explicit computation and storage of the Fréchet derivatives (Leung and Qian, 2006; Plessix, 2006). By comparison, the cost of computing the gradient of the cost function using the adjoint method is on the same order as twice

Manuscript received by the Editor 1 September 2015; revised manuscript received 23 December 2015; published online 27 May 2016.

${ }^{1}$ Formerly King Abdullah University of Science and Technology, Physical Sciences and Engineering Division, Thuwal, Saudi Arabia; presently Princeton University, Department of Geosciences, Princeton, New Jersey, USA. E-mail: uwaheed@princeton.edu.

${ }^{2}$ Schlumberger, Houston, Texas, USA. E-mail: gflagg@ slb.com.

${ }^{3}$ Schlumberger Cambridge Research Center, Cambridge, UK. E-mail: cyarman@slb.com.

(C) 2016 Society of Exploration Geophysicists. All rights reserved. 
the cost of the forward problem (Taillandier et al., 2009). The amount of memory required is governed by the size of the discretized model space and is independent of the available data. Another advantage of the adjoint-state method is that the gradient calculation can be carried out shot-by-shot; therefore, the adjoint-state computation can be distributed over many processors. Despite these attractive features of the adjoint-state method for FAT tomography, its development has been limited to isotropic medium due to unavailability of efficient eikonal solvers for anisotropic media. The recent work of Waheed et al. (2014, 2015a) on efficient anisotropic eikonal solvers has made it possible to extend the adjoint-state method to the anisotropic case (Waheed and Alkhalifah, 2015).

The advent of prestack depth migration demonstrated the need for accurate representation of the subsurface and, hence, the necessity for incorporation of anisotropy into the seismic processing workflow. Furthermore, as data acquisition is progressing toward longer and longer offsets, anisotropy is more pronounced in the measurements. However, inverting for multiple parameters further complicates the already nonlinear inversion process due to the inherent trade-off between parameters (Alkhalifah and Plessix, 2014).

P-wave kinematics in vertical transversely isotropic (VTI) medium is controlled predominantly by three parameters (Alkhalifah, 2011). A successful inversion requires an appropriate choice of these parameters to limit the trade-off between the parameters and to reduce the null space of the inversion procedure. For optimal parameter resolution, we characterize a VTI medium through the NMO velocity $v_{\mathrm{NMO}}$, the anellipticity anisotropy parameter $\eta$, and the Thomsen's anisotropy parameter $\delta$, as suggested by Stopin and Plessix (2014).

In this paper, we use the adjoint-state method for FAT tomography in VTI media. As suggested by earlier studies, $\delta$ is weakly resolvable from surface seismic alone (Plessix and Cao, 2011); therefore, we invert for $v_{\mathrm{NMO}}$ and $\eta$. Our framework enables incorporation of borehole measurements into the adjoint-state formulation. This adds the information needed to resolve the $\delta$ parameter. The rest of the paper is organized as follows: First, we develop theoretical framework for adjoint-state method in VTI media. This is followed by a discussion on issues pertaining to the implementation of the method. Then, we highlight the challenge associated with inverting multiple parameters and address possible solutions. Finally, we test the algorithm on a synthetic benchmark model to demonstrate the accuracy achievable by the algorithm.

\section{THEORY}

FAT tomography aims to find the medium parameters that minimize the difference between the observed and modeled traveltimes. The difference is quantified by a cost function, which we choose to be the least-squares misfit function $J$ given the model parameters $\mathbf{m}$. For a single shot located at $\mathbf{s}$, we define the misfit function by

$$
J(\mathbf{m})=\frac{1}{2} \int_{\partial \Omega}\left|T^{\mathrm{comp}}(\mathbf{m}, \mathbf{r})-T^{\mathrm{obs}}(\mathbf{r})\right|^{2} \mathrm{~d} \mathbf{r},
$$

where the integration is taken over receivers $\mathbf{r}$ located on the acquisition surface $\partial \Omega . T^{\mathrm{comp}}(\mathbf{m}, \mathbf{r})$ and $T^{\mathrm{obs}}(\mathbf{r})$ denote the FAT computed for a particular model $\mathbf{m}$ and the observed traveltimes from the measurements, respectively.

\section{Eikonal solver}

Throughout the remainder of our derivation, we only consider the case of a 2D VTI earth model. The domain of inversion is denoted by $\Omega \subset \mathbb{R}^{2}$, and an arbitrary point in $\Omega$ is denoted by $\mathbf{x}=(x, z)$ $\in \mathbb{R}^{2}$, where $x$ and $z$ are intended to denote the usual lateral and depth oriented coordinates, respectively. Sources and receivers are denoted by $\mathbf{s}, \mathbf{r} \in \mathbb{R}^{2}$, respectively. Our approach is not restricted to this particular case, but for ease of presentation, and the purposes of an initial study of anisotropic FAT tomography, we believe it is sufficient to first consider the $2 \mathrm{D}$ case in detail. The derivation of the adjoint-state method for the full 3D tilted orthorhombic case proceeds in precisely the same manner as the derivation we present here, and thus our method is readily generalizable to higher order symmetries (Flagg and Yarman, 2014).

We parameterize our VTI model with the pseudoacoustic velocity parameters $v_{\mathrm{NMO}}(\mathbf{x}), \eta(\mathbf{x})$, and $\delta(\mathbf{x})$. Here, $v_{\mathrm{NMO}}$ is the NMO velocity, $\eta$ is the anellipticity anisotropy parameter, and $\delta$ is Thomsen's anisotropy parameters (Alkhalifah, 2011). Under this parameterization, the FATs satisfy the acoustic VTI eikonal equation:

$$
\begin{gathered}
v_{\mathrm{NMO}}^{2}(1+2 \eta)\left(\frac{\partial T}{\partial x}\right)^{2}+\frac{v_{\mathrm{NMO}}^{2}}{1+2 \delta}\left(\frac{\partial T}{\partial z}\right)^{2} \\
\times\left(1-2 \eta v_{\mathrm{NMO}}^{2}\left(\frac{\partial T}{\partial x}\right)^{2}\right)=1
\end{gathered}
$$

with the point source condition

$$
T(\mathbf{s})=0,
$$

where $T(\mathbf{x})$ is the traveltime from a source $\mathbf{s}$ to a point $\mathbf{x}$ in the subsurface $\Omega$.

The solution of equation 2 can be computed efficiently by fast sweeping methods (Zhao, 2005). Specifically, we use an iterative fast sweeping-based algorithm to solve the acoustic anisotropic eikonal equation. The details of the algorithm can be found in Waheed et al. (2015b).

\section{Adjoint-state method}

The adjoint-state method computes the gradient of the misfit function. The state variable is traveltime $T$, and the solution of the VTI eikonal equation provides the link between $T$ and the unknown model $\mathbf{m}=\left(v_{\mathrm{NMO}}, \eta, \delta\right)$. The adjoint-state variables are introduced through the Lagrangian formulation and of the augmented misfit functional:

$$
\begin{aligned}
\mathcal{L}\left(v_{\mathrm{NMO}}, \eta, \delta, T, \lambda\right)= & \frac{1}{2} \int_{\partial \Omega}\left|T(\mathbf{r})-T^{\mathrm{obs}}(\mathbf{r})\right|^{2} \mathrm{~d} \mathbf{r} \\
& -\frac{1}{2} \int_{\Omega} \lambda(\mathbf{x}) F\left(v_{\mathrm{NMO}}, \eta, \delta, T\right) \mathrm{d} \mathbf{x}
\end{aligned}
$$

where $\lambda$ represents the adjoint-state variable and 


$$
\begin{aligned}
& F\left(v_{\mathrm{NMO}}, \eta, \delta, T\right)=v_{\mathrm{NMO}}^{2}(1+2 \eta)\left(\frac{\partial T}{\partial x}\right)^{2} \\
& \quad+\frac{v_{\mathrm{NMO}}^{2}}{1+2 \delta}\left(\frac{\partial T}{\partial z}\right)^{2}\left(1-2 \eta v_{\mathrm{NMO}}^{2}\left(\frac{\partial T}{\partial x}\right)^{2}\right)-1 .
\end{aligned}
$$

A perturbation $\delta T=\epsilon \tilde{T}$ in the state variable yields a perturbation $\delta F$ of the form

$$
\delta F=2 \epsilon L(\mathrm{x})^{\top} \nabla \tilde{T}+\mathcal{O}\left(\epsilon^{2}\right),
$$

where $(.)^{\top}$ denotes the transpose operation and

$$
L(\mathbf{x})=\left[\begin{array}{c}
\left(v_{\mathrm{NMO}}^{2}(1+2 \eta)-2 \eta \frac{v_{\mathrm{NMO}}^{4}}{1+2 \delta}\left(\frac{\partial T}{\partial z}\right)^{2}\right) \frac{\partial T}{\partial x} \\
\left(\frac{v_{\mathrm{N}}^{2}}{1+2 \delta}-2 \eta \frac{v_{\mathrm{NMO}}^{4}}{1+2 \delta}\left(\frac{\partial T}{\partial x}\right)^{2}\right) \frac{\partial T}{\partial z}
\end{array}\right] .
$$

We obtain the adjoint-state equations by enforcing

$$
\delta \mathcal{L}=0 .
$$

Expanding the perturbation in the functional $\mathcal{L}$, we obtain

$$
\delta \mathcal{L}=\int_{\partial \Omega}\left(T(\mathbf{r})-T^{\mathrm{obs}}(\mathbf{r})\right) d \mathbf{r}-\int_{\Omega} \lambda(\mathbf{x}) L(\mathbf{x})^{T} \nabla \tilde{T} \mathrm{~d} \mathbf{x} .
$$

Applying the divergence theorem to the last term in the above equation, we get

$$
\begin{aligned}
\delta \mathcal{L} & =\epsilon \int_{\partial \Omega}\left(\left(T(\mathbf{r})-T^{\mathrm{obs}}(\mathbf{r})\right)-\mathbf{n} \cdot(L(\mathbf{x}) \lambda(\mathbf{x}))\right) \tilde{T} \mathrm{~d} \mathbf{r} \\
& +\epsilon \int_{\Omega} \nabla \cdot(L(\mathbf{x}) \lambda(\mathbf{x})) \tilde{T} \mathrm{~d} \mathbf{x}+\mathcal{O}\left(\epsilon^{2}\right)
\end{aligned}
$$

where $\mathbf{n}$ is the unit outward normal of the boundary. To satisfy equation 8 , we require that the two terms in equation 10 are null. Therefore, on the surface, $\lambda$ is the solution of

$$
\text { n. }(L(\mathbf{r}) \lambda(\mathbf{r}))=T^{\mathrm{comp}}(\mathbf{r})-T^{\mathrm{obs}}(\mathbf{r}),
$$

and within the subsurface, $\lambda$ is obtained by solving

$$
\nabla \cdot(L(\mathbf{x}) \lambda(\mathbf{x}))=0 .
$$

The vector $L(\mathbf{x})$ is given in equation 7 . First, the residuals $T^{\mathrm{comp}}(\mathbf{r})-T^{\mathrm{obs}}(\mathbf{r})$ are obtained by solving the forward problem. Then, the adjoint variable is initialized according to equation 11 using the obtained residuals. Then, $\lambda$ is back propagated in the current model to the source position. The equations to solve for adjointstate variable $\lambda$ in presence of borehole measurements are given in Appendix A.

Next, we obtain the gradient of the misfit function $J$ with respect to the model parameters. First, observe that

$$
\delta_{v_{\mathrm{NMO}}} J=\int_{\Omega} \lambda \delta_{v_{\mathrm{NMO}}} F \mathrm{~d} \mathbf{x} .
$$

The perturbation of $F$ with respect to $v_{\mathrm{NMO}}$ is given by

$$
\begin{aligned}
\delta_{v_{\mathrm{NMO}}} F= & 2 \epsilon v_{\mathrm{NMO}} \tilde{v}_{\mathrm{NMO}}(1+2 \eta)\left(\frac{\partial T}{\partial x}\right)^{2} \\
& +\frac{2 \epsilon v_{\mathrm{NMO}} \tilde{v}_{\mathrm{NMO}}}{1+2 \delta}\left(\frac{\partial T}{\partial z}\right)^{2}+\mathcal{O}\left(\epsilon^{2}\right), \\
= & 2 \epsilon \tilde{v}_{\mathrm{NMO}} v_{\mathrm{NMO}}\left((1+2 \eta)\left(\frac{\partial T}{\partial x}\right)^{2}+\frac{1}{1+2 \delta}\left(\frac{\partial T}{\partial z}\right)^{2}\right),
\end{aligned}
$$

where $\epsilon \tilde{v}_{\mathrm{NMO}}$ is a small perturbation to $v_{\mathrm{NMO}}$.

Therefore, we obtain the Fréchet derivative of $J$ with respect to $v_{\mathrm{NMO}}$ as the linear operator $\left(\partial J / \partial v_{\mathrm{NMO}}\right): \delta v_{\mathrm{NMO}} \rightarrow \delta J$ given by

$$
\begin{aligned}
\frac{\partial J}{\partial v_{\mathrm{NMO}}}\left[\delta v_{\mathrm{NMO}}\right]= & \int_{\Omega} v_{\mathrm{NMO}}\left((1+2 \eta)\left(\frac{\partial T}{\partial x}\right)^{2}\right. \\
& \left.+\frac{1}{1+2 \delta}\left(\frac{\partial T}{\partial z}\right)^{2}\right) \lambda \delta v_{\mathrm{NMO}} \mathrm{d} \mathbf{x} .
\end{aligned}
$$

By a similar procedure, we obtain

$$
\frac{\partial J}{\partial \eta}=\int_{\Omega} v_{\mathrm{NMO}}^{2}\left(\frac{\partial T}{\partial x}\right)^{2}\left(1-\frac{v_{\mathrm{NMO}}^{2}}{1+2 \delta}\left(\frac{\partial T}{\partial z}\right)^{2}\right) \lambda \delta \eta \mathrm{d} \mathbf{x} .
$$

Once the gradient of the misfit function $\nabla J$ is computed, we can use a gradient method or equivalent to update the model parameters

$$
\mathbf{m}^{k+1}=\mathbf{m}^{k}-\alpha^{k} \nabla J\left(\mathbf{m}^{k}\right),
$$

where the step length $\alpha^{k}$ is a positive scalar.

\section{Solving for the adjoint-state variable}

Here, we describe a fast sweeping method needed to solve for the adjoint-state variable $\lambda$. The algorithm is adapted from the one developed for the isotropic case by Leung and Qian (2006).

In $2 \mathrm{D}$, the adjoint-state equation 12 can be rewritten as

$$
\frac{\partial}{\partial x}(\alpha a \lambda)+\frac{\partial}{\partial z}(\beta b \lambda)=0,
$$

where

$$
\begin{aligned}
& a=\frac{\partial T}{\partial x}, \quad \alpha=v_{\mathrm{NMO}}^{2}(1+2 \eta)-2 \eta \frac{v_{\mathrm{NMO}}^{4}}{1+2 \delta}\left(\frac{\partial T}{\partial z}\right)^{2}, \\
& b=\frac{\partial T}{\partial z}, \quad \beta=\frac{v_{\mathrm{NMO}}^{2}}{1+2 \delta}-2 \eta \frac{v_{\mathrm{NMO}}^{4}}{1+2 \delta}\left(\frac{\partial T}{\partial x}\right)^{2} .
\end{aligned}
$$

For a computational cell centered at $\left(x_{i}, z_{j}\right)$, discretizing equation 18 using a first-order finite-difference approximation, in the conservative form yields

$$
\begin{aligned}
& \frac{\alpha_{i+\frac{1}{2}, j} a_{i+\frac{1}{2}, j} \lambda_{i+\frac{1}{2}, j}-\alpha_{i-\frac{1}{2}, j} a_{i-\frac{1}{2}, j} \lambda_{i-\frac{1}{2}, j}}{\Delta x} \\
& +\frac{\beta_{i, j+\frac{1}{2}} b_{i, j+\frac{1}{2}} \lambda_{i, j+\frac{1}{2}}-\beta_{i, j-\frac{1}{2}} b_{i, j-\frac{1}{2}} \lambda_{i, j-\frac{1}{2}}}{\Delta z}=0,
\end{aligned}
$$


where $\Delta x$ and $\Delta z$ are the grid spacing in the $x$ - and $z$-directions, respectively. The values of $\lambda$ on grid interfaces $\lambda_{i \pm(1 / 2), j}$ and $\lambda_{i, j \pm(1 / 2)}$ are determined according to the propagation of characteristics. For example, in the case when $a_{i+(1 / 2), j}>0$, the characteristics for determining $\lambda$ goes from the left side of the interface to the right side. This means that we use the value $\lambda_{i, j}$ to define $\lambda_{i+(1 / 2), j}$. Otherwise, we use $\lambda_{i+1, j}$ to define $\lambda_{i+(1 / 2), j}$. The same strategy is applied to obtain values of $\alpha$ and $\beta$ on the interfaces.

Let us introduce the following notations:

$\begin{array}{ll}a_{i+\frac{1}{2}, j}^{ \pm}=\frac{a_{i+\frac{1}{2}, j} \pm\left|a_{i+\frac{1}{2}, j}\right|}{2}, & a_{i-\frac{1}{2}, j}^{ \pm}=\frac{a_{i-\frac{1}{2}, j} \pm\left|a_{i-\frac{1}{2}, j}\right|}{2}, \\ b_{i, j+\frac{1}{2}}^{ \pm}=\frac{b_{i, j+\frac{1}{2}} \pm\left|b_{i, j+\frac{1}{2}}\right|}{2}, & b_{i, j-\frac{1}{2}}^{ \pm}=\frac{b_{i, j-\frac{1}{2}} \pm\left|b_{i, j-\frac{1}{2}}\right|}{2} .\end{array}$

Then, equation 20 becomes

$$
\begin{aligned}
& \frac{\left(a_{i+\frac{1}{2}, j}^{+} \alpha_{i, j} \lambda_{i, j}+a_{i+\frac{1}{2}, j}^{-} \alpha_{i+1, j} \lambda_{i+1, j}\right)-\left(a_{i-\frac{1}{2}, j}^{+} \alpha_{i-1, j} \lambda_{i-1, j}+a_{i-\frac{1}{2}, j}^{-} \alpha_{i, j} \lambda_{i, j}\right)}{\Delta x} \\
& +\frac{\left(b_{i, j+\frac{1}{2}}^{+} \beta_{i, j} \lambda_{i, j}+b_{i, j+\frac{1}{2}}^{-} \beta_{i, j+1} \lambda_{i, j+1}\right)-\left(b_{i, j-\frac{1}{2}}^{+} \beta_{i, j-1} \lambda_{i, j-1}+b_{i, j-\frac{1}{2}}^{-} \beta_{i, j} \lambda_{i, j}\right)}{\Delta z}=0,
\end{aligned}
$$

which can be rewritten as

$$
\begin{gathered}
\left(\alpha_{i, j} \frac{a_{i+\frac{1}{2}, j}^{+}-a_{i-\frac{1}{2}, j}^{-}}{\Delta x}+\beta_{i, j} \frac{b_{i, j+\frac{1}{2}}^{+}-b_{i, j-\frac{1}{2}}^{-}}{\Delta z}\right) \lambda_{i, j} \\
=\frac{a_{i-\frac{1}{2}, j}^{+} \alpha_{i-1, j} \lambda_{i-1, j}-a_{i+\frac{1}{2}, j}^{-} \alpha_{i+1, j} \lambda_{i+1, j}}{\Delta x} \\
+\frac{b_{i, j-\frac{1}{2}}^{+} \beta_{i, j-1} \lambda_{i, j-1}-b_{i, j+\frac{1}{2}}^{-} \beta_{i, j+1} \lambda_{i, j+1}}{\Delta z} .
\end{gathered}
$$

This equation expresses $\lambda_{i, j}$ as a function of its neighboring values $\lambda_{i \pm 1, j}$ and $\lambda_{i, j \pm 1}$. The values of $a$ and $b$ need to be specified at the cell interfaces $\left(x_{i \pm 1}, z_{j}\right)$ and $\left(x_{i}, z_{j \pm 1}\right)$ as per the following relations:

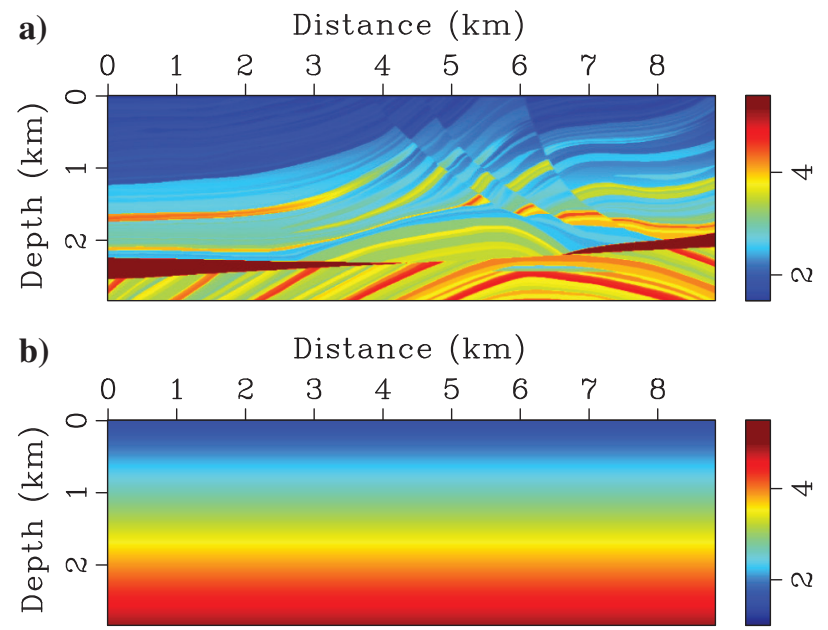

Figure 1. Isotropic velocity for (a) the Marmousi model and (b) the starting model used to compute the gradient.

$$
\begin{array}{rlrl}
a_{i+\frac{1}{2}, j}= & \frac{T_{i+1, j}-T_{i, j}}{\Delta x}, & a_{i-\frac{1}{2}, j}=\frac{T_{i, j}-T_{i-1, j}}{\Delta x}, \\
b_{i, j+\frac{1}{2}}=\frac{T_{i, j+1}-T_{i, j}}{\Delta z}, & b_{i, j-\frac{1}{2}}=\frac{T_{i, j}-T_{i, j-1}}{\Delta z} .
\end{array}
$$

We design a new fast sweeping method to solve equation 23 . The algorithm is similar to the one described in Leung and Qian (2006) to solve the adjoint equation for isotropic media.

\section{IMPLEMENTATION DETAILS}

In this section, we will address practical issues pertaining to implementation of the adjoint-state formulation described in the previous section.

\section{Smoothing the gradient}

The gradient of the misfit function $\nabla J$ is key in driving the optimization algorithm toward the global minima. However, the gradient obtained by numerically solving equations 15 and 16 needs smoothing before being used to update the model. The misfit function is highly nonlinear in nature, particularly when anisotropy is taken into account. Smoothing aims to prevent the algorithm from getting trapped in one of the many local minima (Bording et al., 1987; Bunks et al., 1995; Woodward et al., 2008). In this regard, we begin with a large filter length and gradually reduce it. This is a form of preconditioner we use to help with the convergence of our algorithm.

Another reason to smooth the gradient is to prevent against the strong source signature present in the computed gradient. To illustrate this point, consider an isotropic Marmousi model shown in Figure 1a. The model is $8.8 \mathrm{~km}$ wide and $2.88 \mathrm{~km}$ in depth. A grid spacing of $12 \mathrm{~m}$ is used in both directions. The starting velocity model is a $v(z)$ model as shown in Figure $1 \mathrm{~b}$. We consider receivers spread on the surface at a spacing of $12 \mathrm{~m}$ and compute gradients due to 73 sources evenly spread over the surface. Finally, we sum the gradients due to different sources to compute the total gradient, as shown in Figure 2a. The presence of source signatures can be easily observed in the computed gradient. Therefore, if the gradient is not smoothed, this will lead the inversion algorithm to nonphysi-
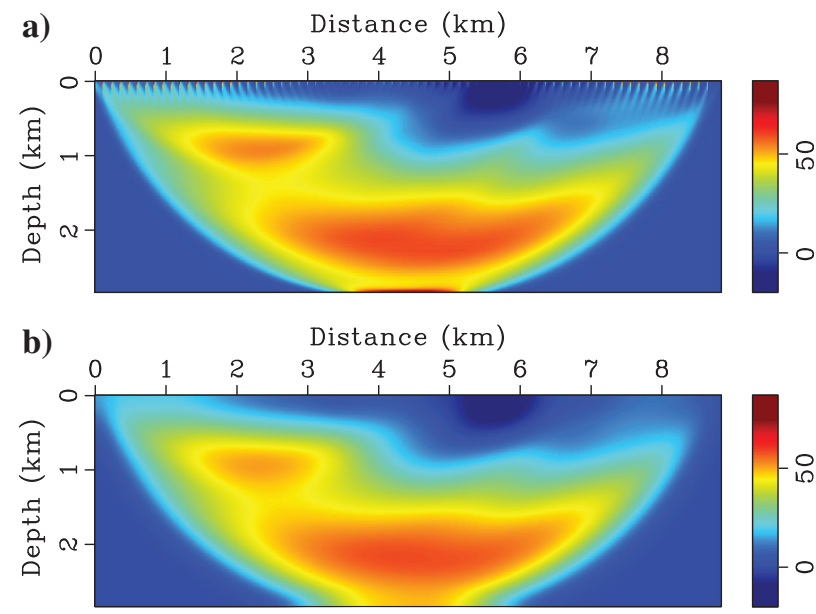

Figure 2. Gradient of the misfit function with respect to velocity (a) before and (b) after triangular smoothing with a filter length of 50 grid points or $1 \mathrm{~km}$ along both dimensions. 
cal models. The gradient after using triangular smoothing with a filter length of 50 grid points or $1 \mathrm{~km}$ along both dimensions is shown in Figure $2 b$.

Considering quality of the inversion result and computational cost, we propose a four stage filtering scheme. Beginning the inversion with a filter length of 50 grid points or $1 \mathrm{~km}$ along both dimensions, we gradually reduce it to the maximum distance between two consecutive sources.

\section{Parallel computation of the gradient}

A major advantage of the adjoint-state method is the ease of parallel implementation. The gradient computation is carried out separately for each shot location before being summed to give the total gradient. These computations are entirely independent from one shot to another, and therefore can be easily distributed across several processors. This results in a significant reduction of the computation time. We parallelize the code using OpenMP. Each processor handles one shot at a time and computes the gradient for it. Once gradient computation for all shots is completed, these gradients are summed to yield the total gradient, as shown in Figure 2a. Because the major cost of tomography lies in gradient computation, parallelization of this step increases the computational speed roughly by a factor equivalent to the number of processors available.

\section{Model update}

Once the gradient of the misfit function is obtained, a local descent optimization method can be used to iteratively update the model and to reduce the misfit. A general form of the update scheme using gradient method is

$$
\mathbf{m}^{k+1}=\mathbf{m}^{k}+\alpha^{k} \boldsymbol{s}^{k},
$$

where $\alpha^{k}$ is the step length to update the model in the search direction $\boldsymbol{s}^{k}$. We use the method of nonlinear conjugate gradient that decreases the misfit along the conjugate gradient direction

$$
\boldsymbol{s}^{k}= \begin{cases}-\nabla J\left(\mathbf{m}^{k}\right), & \text { if } k=0, \\ -\nabla J\left(\mathbf{m}^{k}\right)+\beta^{k} \boldsymbol{s}^{k-1}, & \text { if } k \geq 1 .\end{cases}
$$

Among the several proposed ways to compute $\beta$, we found the Polak-Ribière scheme with automatic reset to have the best convergence properties:

$$
\beta=\max \left\{0, \beta^{\mathrm{PR}}\right\}
$$

where

$$
\beta^{\mathrm{PR}}=\frac{\left(\nabla J\left(\mathbf{m}^{k}\right)\right)^{T}\left(\nabla J\left(\mathbf{m}^{k}\right)-\nabla J\left(\mathbf{m}^{k-1}\right)\right)}{\left(\nabla J\left(\mathbf{m}^{k-1}\right)\right)^{T}\left(\nabla J\left(\mathbf{m}^{k-1}\right)\right)} .
$$

To compute an optimal step length $\alpha$, we need to solve a 1D optimization problem:

$$
\alpha^{k}=\underset{\alpha}{\arg \max } J\left(\mathbf{m}^{k}+\alpha \mathbf{s}^{k}\right) .
$$

A global minimizer of this optimization problem is computationally too expensive to identify. Therefore, we use an inexact line search algorithm satisfying the strong Wolfe criteria, namely the sufficient decrease condition and the curvature condition. A pseudocode for the line search algorithm can be found in Nocedal and Wright (2006).

\section{THE CHALLENGE OF MULTIPARAMETER INVERSION}

The major complication in anisotropic tomography arises due to the need of inverting for multiple parameters. In this regard, the choice of right parameters to characterize the anisotropic medium is essential. This will help in reducing the crosstalk between parameters. We use $v_{\mathrm{NMO}}, \eta$, and $\delta$ to characterize the VTI model. Several studies have pointed at the inherent ambiguity between the depth and the anisotropy parameter (Alkhalifah and Tsvankin, 1995; Plessix and Cao, 2011). Therefore, we keep $\delta$ fixed and invert for $v_{\mathrm{NMO}}$ and $\eta$. This choice of parameters has also been used by Stopin and Plessix (2014), who use it for diving wave FWI. However, Cheng et al. (2014) use vertical velocity $v_{0}$ and $\eta$ for surface seismic FWI. In fact, both choices are equivalent because in both cases, $\delta$ is kept fixed, and the two velocities are related as $v_{\mathrm{NMO}}=v_{0} \sqrt{1+2 \delta}$.

Another difficulty associated with inverting for multiple parameters is the fact that the effects due to different parameters are coupled in the gradient. This creates ambiguity in identifying which parameter contributed to what part of the gradient. To illustrate this point, consider original $v_{\mathrm{NMO}}$ and $\eta$ models as shown in Figure 3. We assume
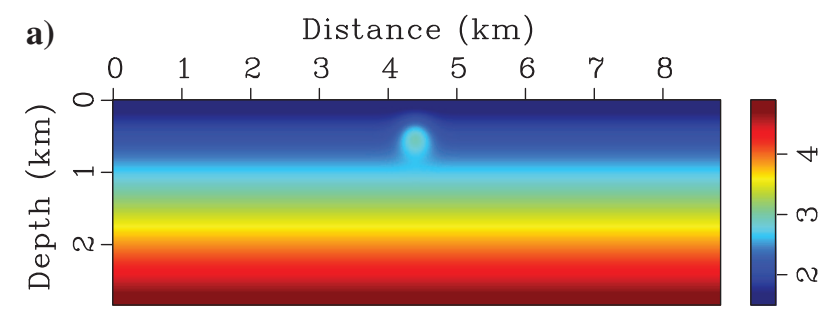

b) Distance $(\mathrm{km})$

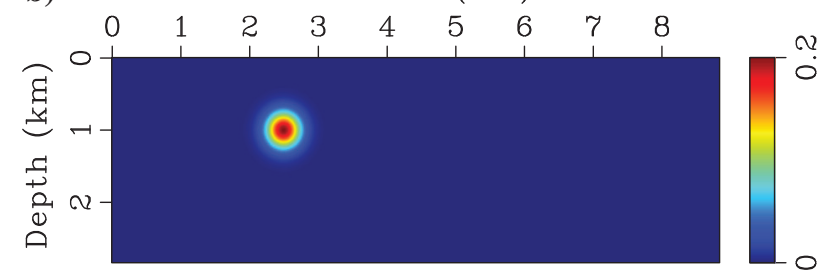

Figure 3. Vertical gradient model for $v_{\mathrm{NMO}}$ with (a) a high-velocity anomaly at $(4.5$ and $0.5 \mathrm{~km})$ and (b) $\eta$ parameter, which is zero everywhere apart from an anomaly at $(2.5$ and $1 \mathrm{~km})$.

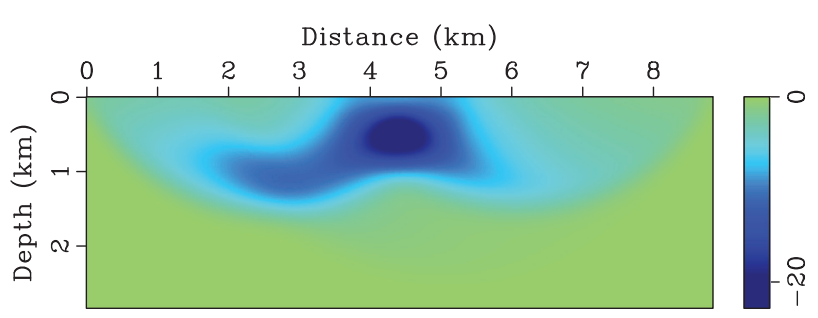

Figure 4. Gradient of the misfit function for the model shown in Figure 3 after smoothing. 
the starting model to be the same as the original model apart from the anomaly.

Using receivers spread on the surface $12 \mathrm{~m}$ apart, we compute the gradient of the misfit function using 73 equispaced sources. The resulting gradient after smoothing is shown in Figure 4. Notice that the gradient captures the presence of anomalies at the correct locations. However, while updating the model, it is ambiguous as to which parameter to update. There is a strong likelihood due to this ambiguity that the updates due to one parameter leaks into the other and vice versa.

We will address our strategy to combat this challenge in the next section.

\section{NUMERICAL TEST}

In this section, we test the anisotropic tomography algorithm to invert for $v_{\mathrm{NMO}}$ and $\eta$ for the VTI SEAM model. The model parameters are shown in Figure 5. We consider part of the original model, which is $40 \mathrm{~km}$ wide and $13.5 \mathrm{~km}$ in depth. We use a total of 101 shots with a shot interval of $400 \mathrm{~m}$. Receivers are spread $20 \mathrm{~m}$ apart on the surface. We use a vertically varying model as a starting model for $v_{\mathrm{NMO}}$. The velocity at the surface is $2 \mathrm{~km} / \mathrm{s}$ and it increases with a gradient of $0.3 \mathrm{~s}^{-1}$. The starting model for $\eta$ is a highly smoothed version of the original $\eta$ model. The smoothing window is $4 \mathrm{~km}$ in both dimensions. We show part of the original and starting $v_{\mathrm{NMO}}$ that is covered by diving waves in Figure $6 \mathrm{a}$ and $6 \mathrm{~b}$, respectively. Similarly, part of the original and starting $\eta$ model that is resolved by diving waves is shown in Figure $7 \mathrm{a}$ and $7 \mathrm{~b}$, respectively.

For anisotropic media, velocity has a first-order effect on the kinematics of wave propagation, whereas the anisotropy parameters
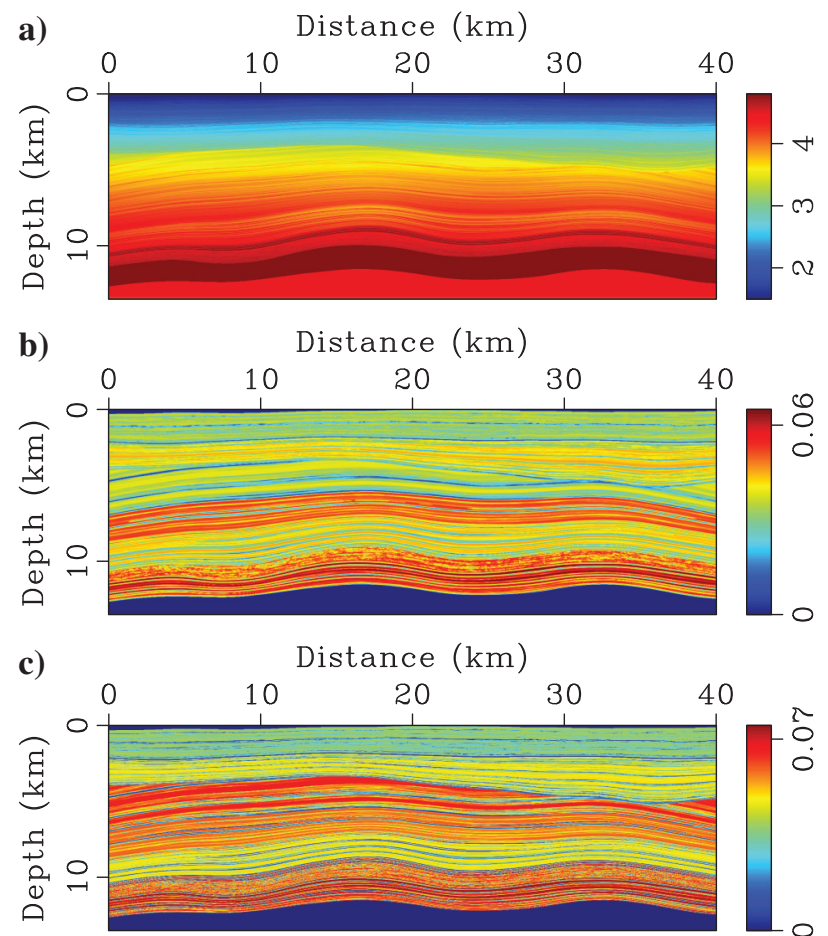

Figure 5. (a) The NMO velocity, (b) the $\eta$ parameter, and (c) the $\delta$ parameter for considered part of the VTI SEAM model.
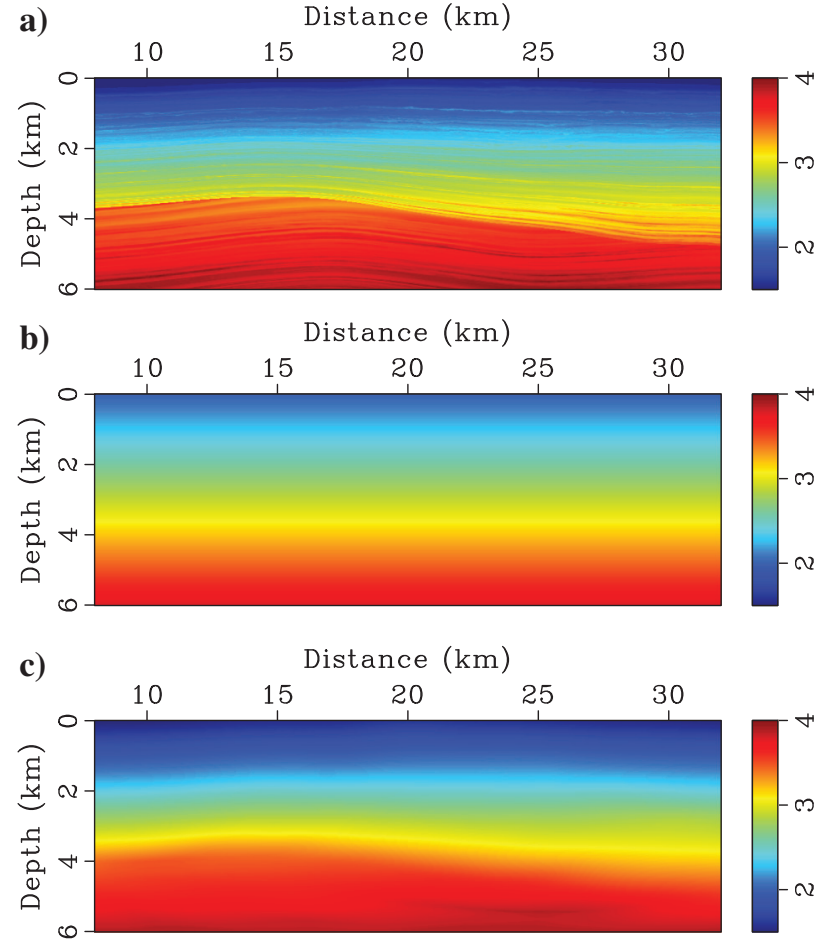

Figure 6. (a) The original $v_{\mathrm{NMO}}$, (b) the starting $v_{\mathrm{NMO}}$, and (c) the inverted $v_{\mathrm{NMO}}$ for part of the VTI SEAM model, resolved by the diving waves.
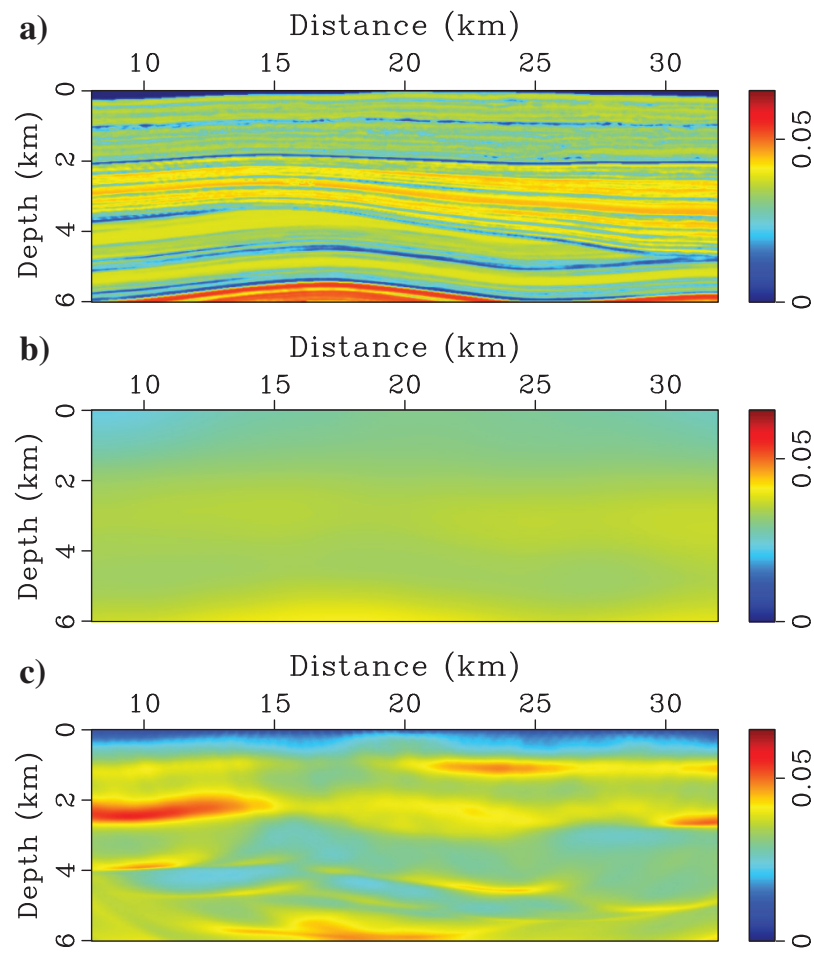

Figure 7. (a) The original $\eta$ model, (b) the starting $\eta$ model, and (c) the inverted $\eta$ model for part of the VTI SEAM model, resolved by the diving waves. 
$\eta$ has a second-order effect. Therefore, we use a hierarchical updating mechanism. First, we begin the inversion by updating $v_{\mathrm{NMO}}$ only until it drives the misfit function close to a minimum. Then, we fix $v_{\mathrm{NMO}}$ and invert for $\eta$. This approach breaks the complicated multiparameter inversion problem into, a more manageable, two single-parameter inversion problems. The inverted $v_{\mathrm{NMO}}$ and $\eta$ parameters are shown in Figures $6 \mathrm{c}$ and $7 \mathrm{c}$, respectively. Comparing Figure $6 a$ and $6 c$, we observe that the long wavelength features of $v_{\mathrm{NMO}}$ have been well-recovered by inversion, particularly the hump on the left part of the model approximately $4 \mathrm{~km}$ deep. Also the macrofeatures in the $\eta$ model are reasonably well-recovered.

Figure 8 compares profiles from the original (red), the starting (magenta), and the inverted (blue) model. For $v_{\mathrm{NMO}}$, the incorrect starting velocities have been corrected significantly to follow the low-frequency trend. For $\eta$, the correction also follows the general trend apart from a few regions, where the update is made opposite to the variation in the original $\eta$ model. This results from the inherent coupling between the parameters. To show convergence history, we consider the residual normalized with respect to the initial residual. A history of the normalized residual is shown in Figure 9. For several source locations, Figure 10 presents the traveltimes observed at the surface (red) and those computed using the starting model (magenta) and the inverted model (blue). Our results show that the traveltimes obtained from the inverted model is in agreement with the data.

As mentioned above, we use a hierarchical update scheme where we first invert for $v_{\mathrm{NMO}}$ only, keeping $\eta$ fixed, and then update $\eta$ after $v_{\mathrm{NMO}}$ has converged to a solution. However, it is not as obvious when to switch between parameters during the inversion due to the coupling between these parameters. A strategy to identify this switching point could use common-image gathers and iteratively correct for the initial guess about the switching point until the gathers are flattened.
We use a fairly simple and cost-efficient procedure to address this problem. The approach assumes knowledge of the upper bound on $\eta$, which can come from a priori knowledge of the geology of the area. First, we force all the update into velocity, saving a few instances of the inverted $v_{\mathrm{NMO}}$; for example, those corresponding to the residual value equal to $10 \%$ and $5 \%$ of the original residual. Next, we use $v_{\mathrm{NMO}}$ obtained when the remaining residual was $10 \%$ of its original value to invert for $\eta$, while thresholding it to the known largest value it can attain. Because $\eta$ is very sensitive to errors in velocity, if the inverted $\eta$ model will exhibit values mostly around its threshold, we observe that updates from velocity are being forced into $\eta$ and take this as an indication that the value of $10 \%$ is early for switching the update parameter. We illustrate

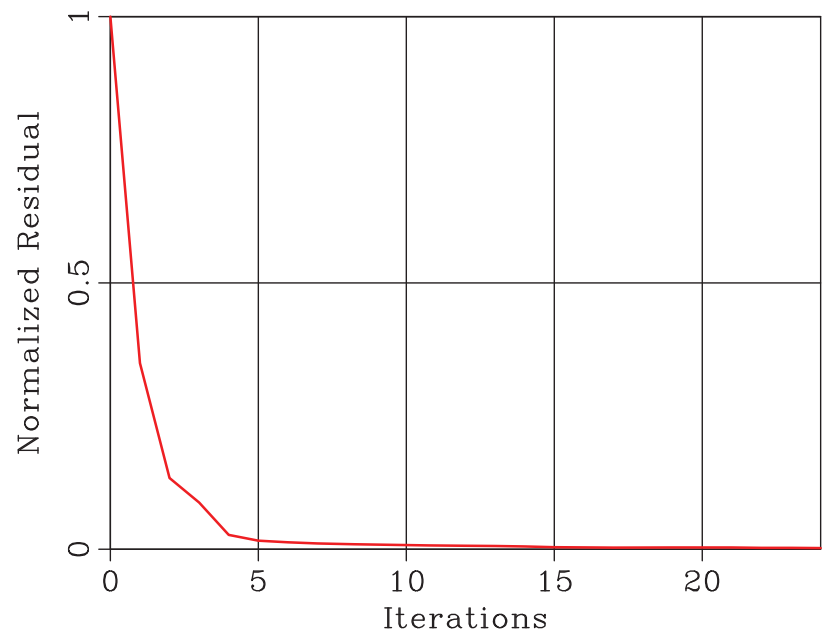

Figure 9. Convergence history for inversion of $v_{\mathrm{NMO}}$ and $\eta$ belonging to the VTI SEAM model.

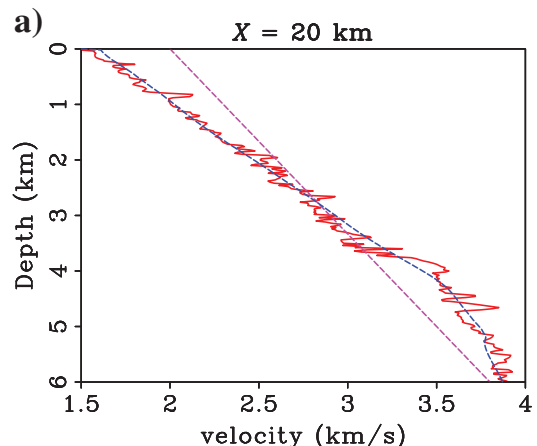

c)
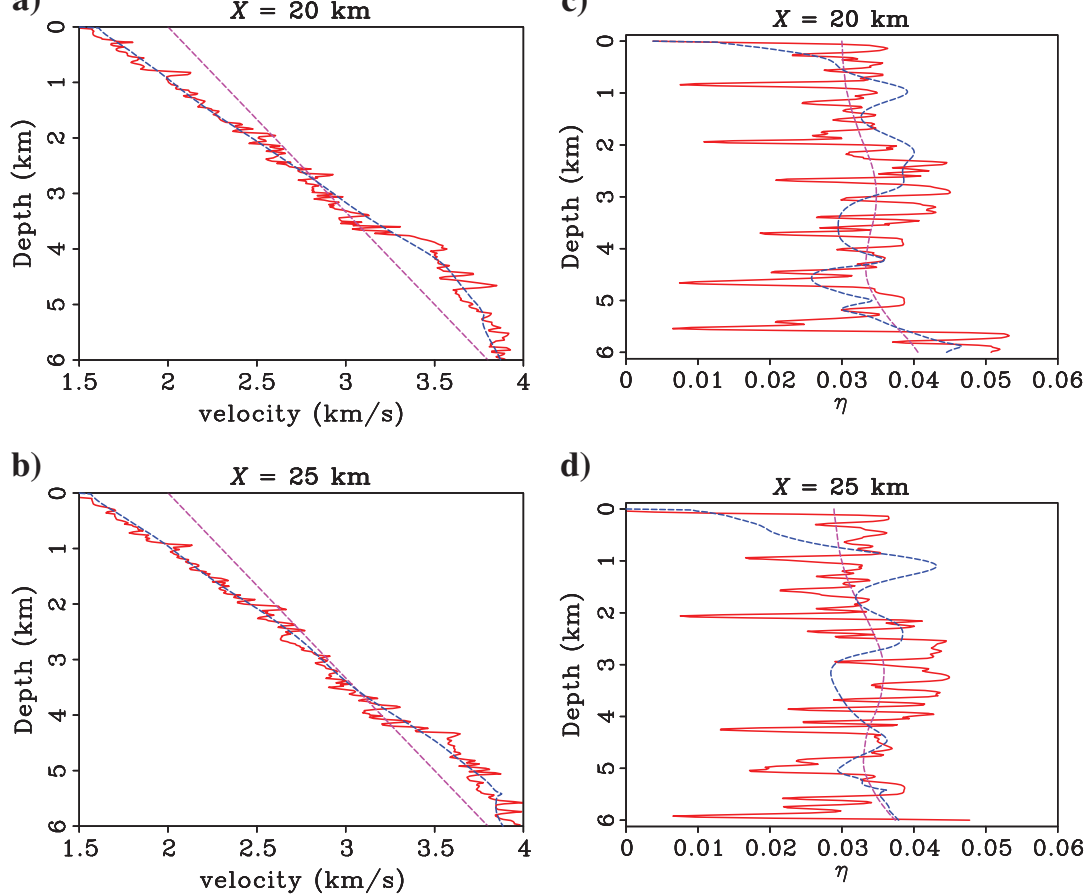

d)

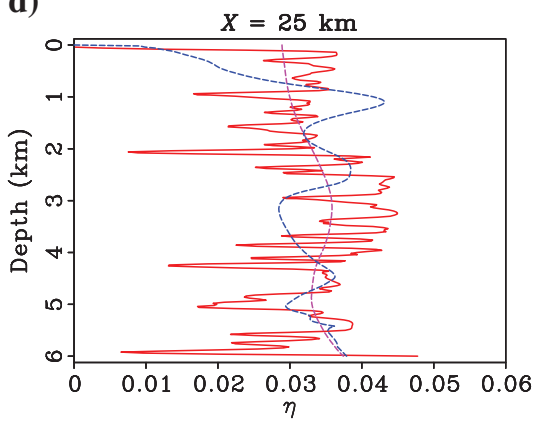

Figure 8. Comparison of profiles for $v_{\mathrm{NMO}}$ at (a) $x=20 \mathrm{~km}$, (b) $x=25 \mathrm{~km}$, and $\eta$ profiles at (c) $x=20 \mathrm{~km}$, and (d) $x=25 \mathrm{~km}$. Red curves show profiles from the original model, the ones from the starting model are plotted in magenta, whereas those from the inverted model are depicted in blue. 
this point in Figure 11, where we plot the inverted $\eta$ obtained by switching from $v_{\mathrm{NMO}}$ to $\eta$.

Next, we consider the $v_{\mathrm{NMO}}$ corresponding to $5 \%$ residual value and use it to invert $\eta$. If the update in $\eta$ will have minor changes compared with the starting $\eta$ model, we take this as a sign that the $\eta$ updates are absorbed in the $v_{\mathrm{NMO}}$ updates. Figure 12 illus-
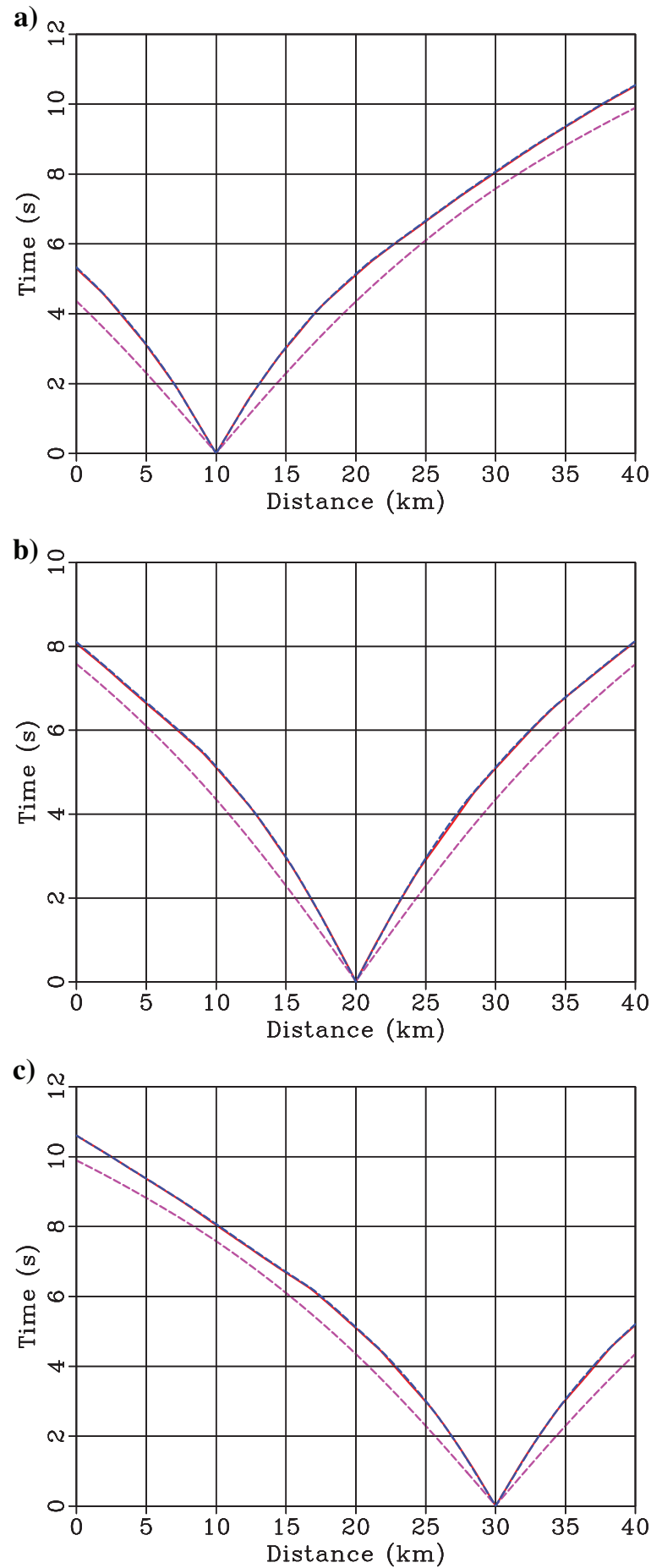

Figure 10. Observed traveltimes at the surface (red) and those computed using the starting model (magenta) and the inverted model (blue) for a source located at: (a) $x=10 \mathrm{~km}$, (b) $x=20 \mathrm{~km}$, and (c) $x=30 \mathrm{~km}$.
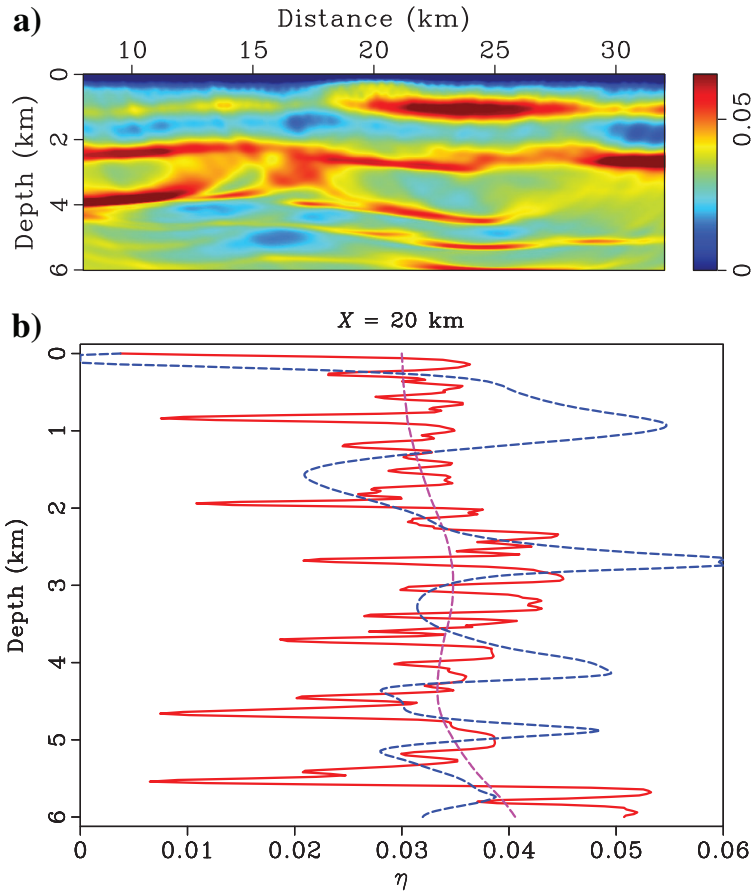

Figure 11. Inverted $\eta$ model, where we switched from $v_{\mathrm{NMO}}$ to $\eta$ before (a) the optimal switching point, and (b) an $\eta$ profile comparing the original (red), the starting (magenta), and the inverted (blue) model for this case. Notice, a lot of $\eta$ values are close to the threshold indicating significant leakage from $v_{\mathrm{NMO}}$.
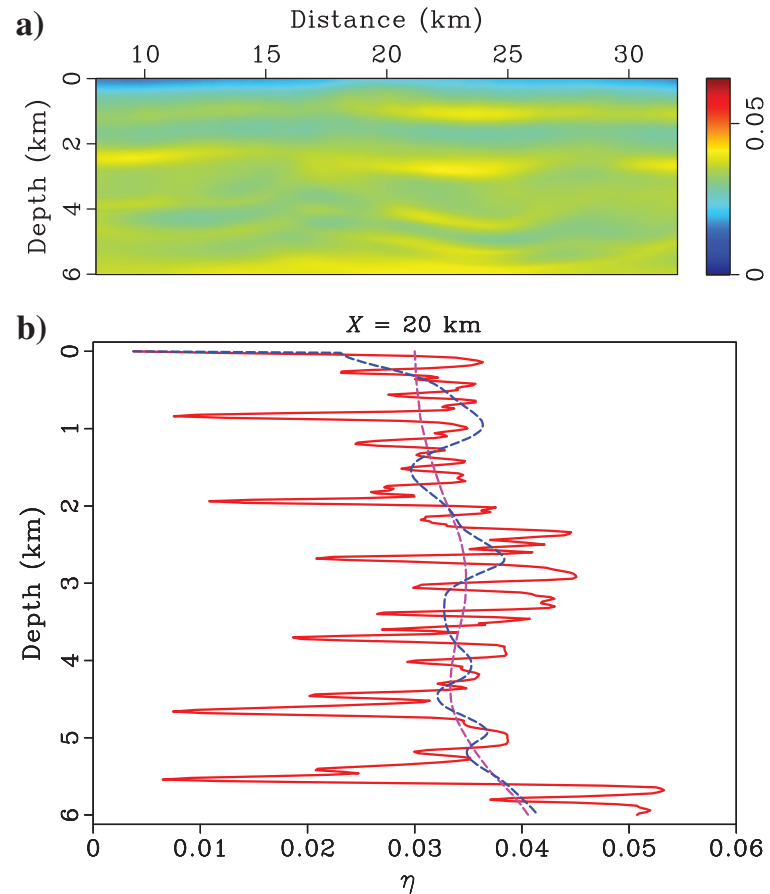

Figure 12. Inverted $\eta$ model, where we switched from $v_{\mathrm{NMO}}$ to $\eta$ after (a) the optimal switching point, and (b) an $\eta$ profile comparing the original (red), the starting (magenta), and the inverted (blue) model for this case. Notice that minor updates were made to the starting $\eta$ model indicating that the optimal switching point had already passed. 
trates this point. This indicates that the point for switching between parameters, corresponding to the least crosstalk between recovered $v_{\mathrm{NMO}}$ and $\eta$, lies between the two residual values. Using this strategy, we obtained the inversion results shown in Figures $6 \mathrm{c}$ and $7 \mathrm{c}$.

\section{CONCLUSIONS}

FAT tomography has been used for near-surface modeling, which has been used for static corrections in complex media, as well as an initial model for depth migration and FWI. Determination of nearsurface model parameters is a key step in imaging deeper structures. Incorporation of anisotropy is inevitable for high-end near-surface modeling, microseismic-source localization, and fractured reservoir characterization. A robust, feasible, and efficient FAT tomography method for anisotropic media is a valuable tool for these applications.

In this paper, we extended the adjoint-state method to invert for parameters in a VTI medium. Using a hierarchical scheme, we inverted for $v_{\mathrm{NMO}}$ and $\eta$ parameter. We also develop mathematical framework for incorporating borehole measurements into the adjoint-state technique, which will be crucial in inversion for $\delta$ parameter (Appendix A). The mathematical formulation developed here can be easily extended, in a similar way, to lower anisotropic symmetries, such as orthorhombic. Through synthetic test, we demonstrate accuracy and robustness of the algorithm. The algorithm can also be used for accurate single-parameter inversion, where the information regarding other parameters come from other sources, such as reflection tomography, etc.

\section{ACKNOWLEDGMENTS}

We thank Schlumberger for financial support and permission to publish results. We are also grateful to X. Cheng, M. Woodward, J. Rickett, C. Chapman, and M. Williams for useful discussions. We extend gratitude to K. Innanen, J. Cao, A. Bona, and S. Charles for many useful suggestions that greatly helped in improving the quality of the paper.

\section{APPENDIX A}

\section{INCORPORATION OF BOREHOLE MEASUREMENTS}

The adjoint-state method for FAT tomography (Leung and Qian, 2006) does not address the modifications needed to incorporate borehole measurements into the computation of adjoint-state variable $\lambda$. However, traveltime tomography based on diving waves needs an additional dimension of information, such as boreholes, to uniquely resolve $\delta$ parameter. In case, we have receivers on the surface in addition to boreholes, we first initialize $\lambda$ using equation 11 , and then compute $\lambda$ in the subsurface by solving equation 12 apart from the borehole locations, where we solve

$$
\nabla .(L(\mathbf{x}) \lambda(\mathbf{x}))=T^{\mathrm{comp}}(r)-T^{\mathrm{obs}}(r)
$$

For the case in which we consider receivers only in the borehole, we only solve equations 12 and A-1. An inversion scheme combining the approach presented in the text with borehole measurements for $\delta$ inversion remains a topic of future publications.

\section{REFERENCES}

Alkhalifah, T., 2011, Scanning anisotropy parameters in complex media: Geophysics, 76, no. 2, U13-U22, doi: 10.1190/1.3553015.

Alkhalifah, T., and R.-É. Plessix, 2014, A recipe for practical fullwaveform inversion in anisotropic media: An analytical parameter resolution study: Geophysics, 79, no. 3, R91-R101, doi: 10.1190/ geo2013-0366.1.

Alkhalifah, T., and I. Tsvankin, 1995, Velocity analysis for transversely isotropic media: Geophysics, 60, 1550-1566, doi: 10.1190/1.1443888.

Bergman, B., A. Tryggvason, and C. Juhlin, 2004, High-resolution seismic traveltime tomography incorporating static corrections applied to a tillcovered bedrock environment: Geophysics, 69, 1082-1090, doi: 10 $.1190 / 1.1778250$.

Bording, R. P., A. Gersztenkorn, L. R. Lines, J. A. Scales, and S. Treitel, 1987, Applications of seismic travel-time tomography: Geophysical Journal International, 90, 285-303, doi: 10.1111/j.1365-246X.1987.tb00728.x.

Brenders, A., and R. G. Pratt, 2007, Waveform tomography of marine seismic data: What can limited offset offer: 75th Annual International Meeting, SEG, Expanded Abstracts, 3024-3028.

Bunks, C., F. M. Saleck, S. Zaleski, and G. Chavent, 1995, Multiscale seismic waveform inversion: Geophysics, 60, 1457-1473, doi: 10.1190/1 .1443880 .

Cheng, X., K. Jiao, D. Sun, and D. Vigh, 2014, Multiparameter fullwaveform inversion for acoustic VTI medium with surface seismic data: 76th Annual International Conference and Exhibition, EAGE, Extended Abstracts.

Dessa, J.-X., S. Operto, S. Kodaira, A. Nakanishi, G. Pascal, K. Uhira, and Y. Kaneda, 2004, Deep seismic imaging of the eastern Nankai trough Japan, from multifold ocean bottom seismometer data by combined travel time tomography and prestack depth migration: Journal of Geophysical Research: Solid Earth (1978-2012), 109, B02111, doi: 10.1029/ 2003JB002689.

Flagg, G., and C. Yarman, 2014, Anisotropic first arrival tomography: WG internal communication.

Leung, S., and J. Qian, 2006, An adjoint state method for three-dimensional transmission traveltime tomography using first-arrivals: Communications in Mathematical Sciences, 4, 249-266, doi: 10.4310/CMS.2006.v4.n1. a10.

Marsden, D., 1993, Static corrections - A review, Part 1: The Leading Edge, 12, 43-49, doi: 10.1190/1.1436912.

Nocedal, J., and S. Wright, 2006, Nonlinear optimization: Springer.

Plessix, R.-É., 2006, A review of the adjoint-state method for computing the gradient of a functional with geophysical applications: Geophysica Journal International, 167, 495-503, doi: 10.1111/j.1365-246X.2006 .02978.x.

Plessix, R.-É., and Q. Cao, 2011, A parametrization study for surface seismic full waveform inversion in an acoustic vertical transversely isotropic medium: Geophysical Journal International, 185, 539-556, doi: 10.1111/j 1365-246X.2011.04957.x.

Stopin, A., and R.-É. Plessix, 2014, Land seismic data multi-parameter waveform inversion: 76th Annual International Conference and Exhibition, EAGE, Extended Abstracts.

Taillandier, C., M. Noble, H. Chauris, and H. Calandra, 2009, First-arrival traveltime tomography based on the adjoint-state method: Geophysics, 74, no. 6, WCB1-WCB10, doi: 10.1190/1.3250266.

Virieux, J., and S. Operto, 2009, An overview of full-waveform inversion in exploration geophysics: Geophysics, 74, no. 6, WCC1-WCC26, doi: 10 $.1190 / 1.3238367$.

Waheed, U., and T. Alkhalifah, 2015, Fast sweeping methods for factored TTI eikonal equation: 77th Annual International Conference and Exhibition, EAGE, Extended Abstracts, doi: 10.3997/2214-4609 .201412998.

Waheed, U., T. Alkhalifah, and H. Wang, 2015a, Efficient traveltime solutions of the acoustic TI eikonal equation: Journal of Computational Physics, 282, 62-76, doi: 10.1016/j.jcp.2014.11.006.

Waheed, U., T. Alkhalifah, and H. Wang, 2015b, An iterative, fast-sweeping-based eikonal solver for 3D tilted anisotropic media: Geophysics, 80, no. 3, C49-C58, doi: 10.1190/geo2014-0375.1.

Waheed, U., C. E. Yarman, and G. Flagg, 2014, An iterative fast sweeping based eikonal solver for tilted orthorhombic media: 84th Annual International Meeting, SEG, Expanded Abstracts, 480-485.

Woodward, M. J., D. Nichols, O. Zdraveva, P. Whitfield, and T. Johns, 2008 A decade of tomography: Geophysics, 73, no. 5, VE5-VE11, doi: 10 $.1190 / 1.2969907$.

Zelt, C. A., and P. J. Barton, 1998, Three-dimensional seismic refraction tomography: A comparison of two methods applied to data from the Faeroe Basin: Journal of Geophysical Research: Solid Earth (1978-2012), 103, 7187-7210, doi: 10.1029/97JB03536.

Zhao, H., 2005, A fast sweeping method for eikonal equations: Mathematics of Computation, 74, 603-628, doi: 10.1090/S0025-5718-04-01678-3. 